\title{
Robust Commitments and Partial Reputation
}

\author{
VIDYA MUTHUKUMAR and ANANT SAHAI, Department of EECS, University of California, Berkeley
}

Agents rarely act in isolation - their behavioral history, in particular, is public to others. We seek a nonasymptotic understanding of how a leader agent should shape this history to its maximal advantage, knowing that follower agent(s) will be learning and responding to it. We study Stackelberg leader-follower games with finite observations of the leader commitment, which commonly models security games and network routing in engineering, and persuasion mechanisms in economics. First, we formally show that when the game is not zero-sum and the vanilla Stackelberg commitment is mixed, it is not robust to observational uncertainty. We propose observation-robust, polynomial-time-computable commitment constructions for leader strategies that approximate the Stackelberg payoff, and also show that these commitment rules approximate the maximum obtainable payoff (which could in general be greater than the Stackelberg payoff). Full paper: https://eecs berkeley.edu/ sahai/reputation.pdf

CCS Concepts: • Theory of computation $\rightarrow$ Solution concepts in game theory; Exact and approximate computation of equilibria; Convergence and learning in games.

Additional Key Words and Phrases: Stackelberg, commitment, reputation, polytope, optimization, learning

ACM Reference Format:

Vidya Muthukumar and Anant Sahai. 2019. Robust Commitments and Partial Reputation. In ACM EC '19: ACM Conference on Economics and Computation (EC '19), June 24-28, 2019, Phoenix, AZ, USA. ACM, New York, NY, USA, 2 pages. https://doi.org/10.1145/3328526.3329647

\section{INTRODUCTION}

In several mechanisms in engineering and economics, myopic "follower" agents observe the history of play of an incumbent "leader" agent and respond as strategic entities. Asymptotically, the leader should commit to her Stackelberg strategy to obtain maximal advantage from the interaction. The full power of commitment is known to provide a significant advantage to the leader [3, 4]. In reality, the leader and follower(s) interact only a finite number of times. Thus, followers may not be able to observe the exact nature of the commitment, or even believe in it. We focus on understanding the incentives of a leader whose reputation is being learned, and ask how should she shape her partially realized commitment for maximal advantage.

\section{PROBLEM FORMULATION}

We represent a two-player game in normal form by the pair of $m \times n$ matrices $(A, B)$, where $A=\left[\begin{array}{llll}\mathbf{a}_{1} & \mathbf{a}_{2} & \ldots & \mathbf{a}_{n}\end{array}\right]$ and $B=\left[\begin{array}{llll}\mathbf{b}_{1} & \mathbf{b}_{2} & \ldots & \mathbf{b}_{n}\end{array}\right]$ denote the leader and follower payoff matrices respectively. We assume that the leader knows about the follower preferences (i.e. $B$ ) while the follower does not know about the leader preferences (i.e. A). Let a leader commitment be denoted by any $m$-dimensional mixed strategy $\mathbf{x}$. We denote the follower's best response to the mixed strategy that it observes by $j^{*}(\mathbf{x}) \in[m]$. Note that $j^{*}(\mathbf{x})$ is a pure strategy and an important traditional assumption is that the follower breaks ties in favor of the leader [2]. A leader will expect ideal

Permission to make digital or hard copies of part or all of this work for personal or classroom use is granted without fee provided that copies are not made or distributed for profit or commercial advantage and that copies bear this notice and the full citation on the first page. Copyrights for third-party components of this work must be honored. For all other uses, contact the owner/author(s).

EC '19, June 24-28, 2019, Phoenix, AZ, USA

(C) 2019 Copyright held by the owner/author(s).

ACM ISBN 978-1-4503-6792-9/19/06.

https://doi.org/10.1145/3328526.3329647 
payoff $f_{\infty}(\mathbf{x}):=\left\langle\mathbf{x}, \mathbf{a}_{j^{*}(\mathbf{x})}\right\rangle$ from mixed commitment $\mathbf{x}$. Therefore, the leader's ideal Stackelberg payoff is defined by $f_{\infty}^{*}:=\max _{\mathbf{x}} f_{\infty}(\mathbf{x})$. The argmax is denoted as the Stackelberg commitment $x_{\infty}^{*}$. Formally, a leader can only partially reveal her commitment $\mathbf{x}$ through $N$ pure strategy plays $I_{1}, I_{2}, \ldots, I_{N}$ i.i.d $\sim \mathbf{x}$. The follower computes the MLE estimate of leader commitment, $\widehat{\mathbf{x}}_{N}$. Then, a "rational" follower would play the pure strategy $j^{*}\left(\widehat{\mathbf{x}}_{N}\right)$ and the leader would expect payoff $f_{N}(\mathbf{x}):=\mathbb{E}\left[\left\langle\mathbf{x}, \mathbf{a}_{j^{*}\left(\widehat{\mathbf{x}}_{N}\right)}\right\rangle\right]$. We denote $f_{N}^{*}:=\max _{\mathbf{x}} f_{N}(\mathbf{x})$ and $x_{N}^{*}$ as the argmax. Note that evaluating $f_{N}^{*}$ exactly would involve a non-convex optimization problem.

\section{MAIN RESULTS}

(Non)-robustness of Stackelberg commitments. It was shown in earlier work that the payoff of the Stackelberg commitment is robust to observational uncertainty when the game is zero sum [1]. The story is more complex for non-zero-sum games: it turns out that the follower response is highly stochastic even for tiny amounts of uncertainty, and such different responses lead to a sharp drop in leader payoff. The following theorem shows that this is the norm rather than the exception.

Theorem 3.1. For any non-zero-sum game with a mixed Stackelberg commitment $x_{\infty}^{*}$, and payoff $f_{\infty}(\cdot)$ discontinuous at $x_{\infty}^{*}$, we have $\lim _{N \rightarrow \infty} f_{N}\left(x_{\infty}^{*}\right) \leq f_{\infty}^{*}-C$ for some positive constant $C>0$.

Simple, robust commitment constructions. The linear program characterization of Stackelberg equilibrium ([2]) pushes the commitment $x_{\infty}^{*}$ all the way to the boundary of a best-response-polytope If the leader wanted to improve commitment learnability, she could move her commitment to the interior of the polytope to elicit the expected follower response with high probability. She will want to do this while remaining sufficiently close to the ideally optimal Stackelberg commitment $x_{\infty}^{*}$. Our robust commitment constructions approximately optimize this tradeoff.

THeOREM 3.2. We construct commitments $\left\{\mathbf{x}_{N}\right\}_{N \geq 1}$ for which $f_{\infty}^{*}-f_{N}\left(\mathbf{x}_{N}\right)=\widetilde{O}\left(\sqrt{\frac{1}{N}}\right)$.

Further, we can compute these constructions in constant time from the Stackelberg commitment.

Approximation guarantees on maximum obtainable payoff. The robust commitment constructions optimized follower learnability. However, the leader could also try and fool the follower into responding favorably, which creates the potential for a gain over and above Stackelberg. To show that our robust commitments are approximately optimal, we show that the potential for such gain decreases with $N$ regardless of the choice of commitments.

Theorem 3.3. For any game $(A, B)$, we have $f_{N}^{*} \leq f_{\infty}^{*}+O\left(\sqrt{\frac{n}{N}}\right)$.

Theorems 3.2 and 3.3 together imply that our robust commitments $\left\{f_{N}\left(\mathbf{x}_{N}\right)\right\}_{N \geq 1}$ provide an $O\left(\frac{1}{\sqrt{N}}\right)$-additive approximation to the optimum $f_{N}^{*}$.

\section{ACKNOWLEDGMENTS}

We thank the anonymous reviewers for valuable feedback. We gratefully acknowledge the support of the NSF through grant AST-1444078, and the Berkeley ML4Wireless research center.

\section{REFERENCES}

[1] Avrim Blum, Nika Haghtalab, and Ariel D Procaccia. 2014. Lazy Defenders Are Almost Optimal against Diligent Attackers.. In AAAI. 573-579.

[2] Vincent Conitzer and Tuomas Sandholm. 2006. Computing the optimal strategy to commit to. In Proceedings of the 7th ACM Conference on Electronic Commerce. ACM, 82-90.

[3] Drew Fudenberg and David K Levine. 1989. Reputation and equilibrium selection in games with a patient player. Econometrica: Journal of the Econometric Society (1989), 759-778.

[4] Bernhard Von Stengel and Shmuel Zamir. 2010. Leadership games with convex strategy sets. Games and Economic Behavior 69, 2 (2010), 446-457. 